\title{
Three-Dimensional Cathodoluminescence by Focused Ion Beam - Scanning Electron Microscopy
}

\author{
D.A.M. de Winter ${ }^{1}$, G.M.Pennock ${ }^{2}$, J.A. Post $^{1}$ and M.R. Drury ${ }^{2}$ \\ 1. Biomolecular Imaging, Faculty of Science, Utrecht University, Utrecht, The Netherlands. \\ 2. Department of Earth Sciences, Faculty of Geosciences, Utrecht University, Utrecht, The Netherlands
}

Cathodoluminescence (CL) imaging in a Scanning Electron Microscope (SEM) is the detection of light induced by the electron beam. It is an established technique, for example in visualization of the formation and growth of crystals by mapping trace elements and structural defects. A difference of a few hundred parts per million can already provide contrast in CL imaging, undetectable with conventional SEM imaging techniques.

Standard SEM and SEM-CL imaging of polished surfaces result in two-dimensional information of the three-dimensional structures, such as grain boundary orientations. Obtaining three-dimensional information can be achieved by combining the SEM with a Focused Ion beam (FIB). The FIB enables cutting cross sections at predefined areas and the SEM is subsequently employed for imaging the cross section. The process of consecutive milling and imaging is called FIB-SEM Tomography and results in three-dimensional models of the volume of interest [1]. The combination of CL and FIB-SEM Tomography introduces the third dimension in CL imaging.

Here we present the on-going development of the combination of FIB-SEM Tomography with CL imaging, both theoretically and in practice as initially reported in [2].

As the FIB is known for creating damage close to the surface (e.g. gallium doping, forming selfinterstitials, crystal defect structures) the first question to be addressed is: does the CL emission survive the FIB milling? The depth of the interaction volume for the CL emission in the SEM (at $10-30 \mathrm{kV}$ ) generally exceeds the FIB damage depth. Therefore it is expected that at least a part of the original CL emission will be still generated. In addition to that, in simulations in the literature [3] it is shown that for certain SEM conditions a thin surface defect layer actually amplifies the CL emission. From a theoretical point of view it is concluded that FIB-SEM CL Tomography is feasible.

Our experimental setup consists of a parabolic mirror in between the sample and the SEM pole piece, reflecting the light towards the detector. Unfortunately the mirror blocks the view for the FIB and has to be inserted / retracted manually. Therefore an automated version is not possible at this moment. In addition the stage can't be tilted when the CL detector is in position. To be able to image a FIB cross section with the SEM, milling is performed at zero degrees stage tilt, resulting in an inclined cross section. Although currently laborious, from a practical point of view it is concluded that FIB-SEM CL Tomography is feasible and valuable. Both modes of operation (milling and CL imaging) are depicted in figure 1.

Actual FIB-SEM CL Tomography data has been obtained from a diamond containing growth layers [2] and inclusions [4]. From the series of CL images a three-dimensional reconstruction can be made, where it has to be taken into account that the cross sections are not perpendicular to the surface. 
In addition to growth patterns in diamond, FIB-SEM CL tomography has been applied to ceramic materials, showing the three dimensional topology of grains and the intergranular glass (figure 2). As CL emission is not influenced by charging, CL imaging of the uncoated FIB cross sections is put forward as a solution for imaging grain boundaries and glass, as conventional BackScatter Electron (BSE) emission is severely influenced by charging of the glass. The three dimensional information on glass distribution around the crystals is crucial in understanding the physical properties of these materials.

The combination of FIB-SEM Tomography with BSE imaging and CL imaging brings together complementary imaging modes which together can provide new insights in the microstructures of samples from the fields of Geology and Semiconductors.

\section{References:}

[1] D.A.M. de Winter et al, Journal of Microscopy 223 (3) (2009), p. $372-383$.

[2] D.A.M. de Winter et al, Journal of Microscopy 242 (3) (2011), p. 315 - 326.

[3] A. Nouiri, A. Djemel and R.J. Tarento, Microelectronic Engineering 51-52 (2000), p. 151 - 156.

[4] De Vries et al, Contributions to Mineralogy and Petrology 161 (40) (2011), p 565 - 579.

[5] This research is financially supported by the Netherlands research school for Integrated Solid Earth Science and the Dutch NWO Groot funding.
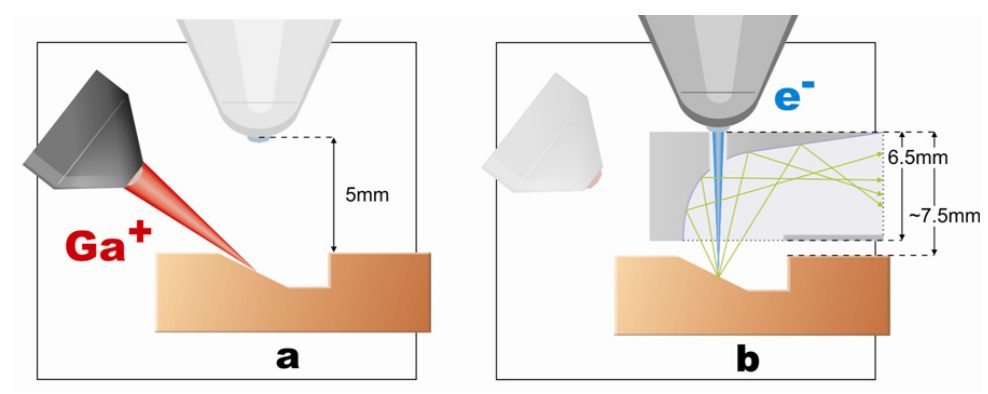

Figure 1. The two modes of operation. (a) The Focused Ion Beam (FIB) mills an inclined cross section allowing (b) Cathodoluminescence (CL) imaging.
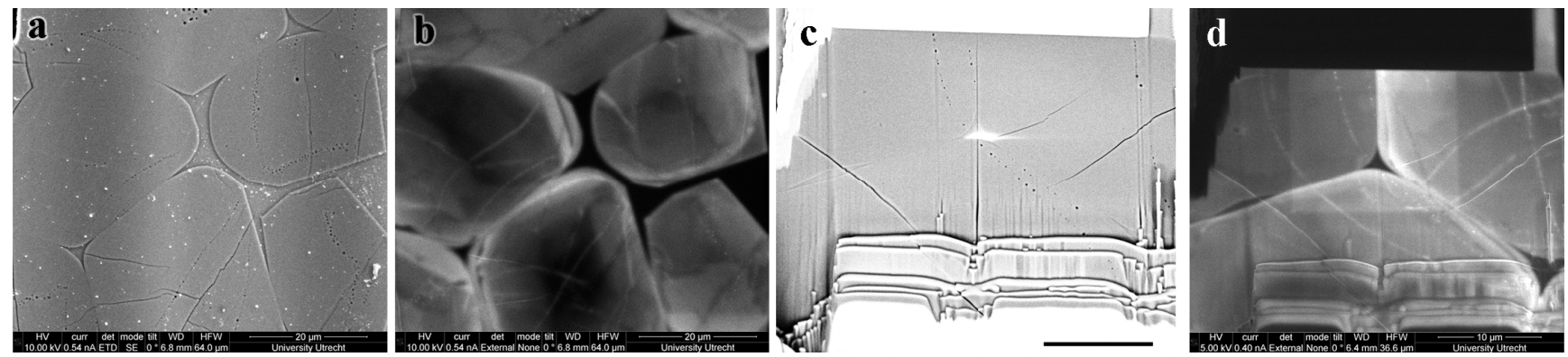

Figure 2. (a) A Secondary Electron and (b) a Cathodoluminescence (CL) image of a mechanically polished olivine $\left((\mathrm{Mg}, \mathrm{Fe})_{2} \mathrm{SiO}_{4}\right)$ surface. (c) A BackScatter Electron image from a FIB cross section (scale bar $10 \mu \mathrm{m}$ ) and (d) the corresponding CL image. 